\title{
Julius Beischer feiert seinen 70. Geburtstag
}

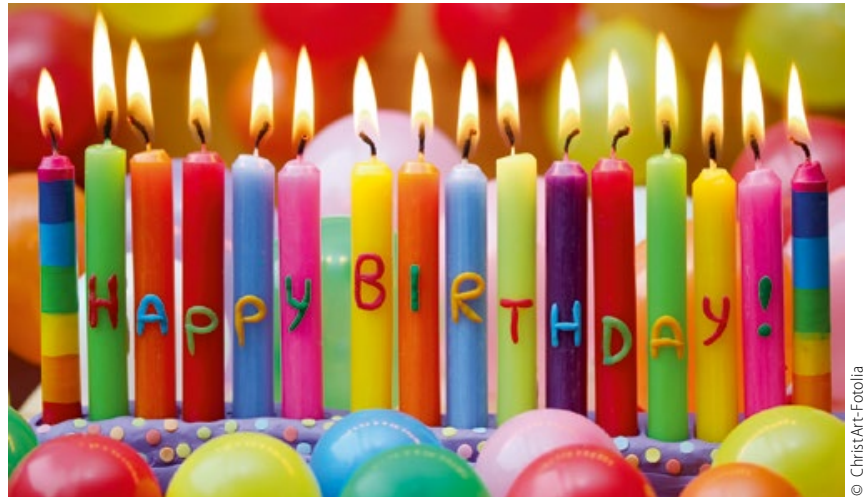

Am 14. November 1946 in Bayern geboren, landete Dr. Julius Beischer nach entbehrungsreicher Jugend im Rheinland, wurde Industriekaufmann bei Thyssen und holte schließlich auf der Darmstädter Marienhöhe sein Abitur nach. Bereits da bewies er erste journalistische Fähigkeiten bei der Gestaltung und Herausgabe eines von ihm konzipierten und gestalteten Schuljahrbuchs. Zahlreiche Aushilfsjobs in verschiedenen Branchen finanzierten die lehr- und erfahrungsreiche Schulzeit.

Inspiriert vom eigenen Bruder wurde Beischer jedoch nicht Journalist, sondern begann das Studium der Zahnmedizin in Hannover. Den Griffel ließ er allerdings nicht fallen - im Gegenteil: Der Zahnmedizinstudent wurde der erste Chefredak- teur des von ihm ins Leben gerufenen studentischen ZM-Infos. Damit nahm er den journalistischen Faden wieder auf. Als Fachschaftssprecher stellte er zudem seine Eignung für spätere berufspolitische Ämter unter Beweis.

1984 folgte die Gründung einer immer noch aktiven Gemein-

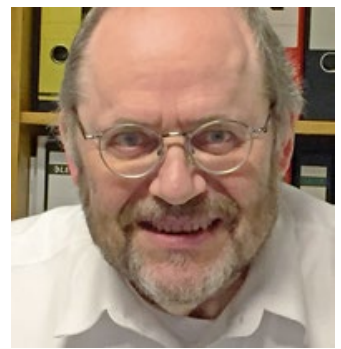
schaftspraxis in Bad Fallingbostel in der Lüneburger Heide und ein Jahr später der Eintritt in den Freien Verband Deutscher Zahnärzte (FVDZ), in dem er zahlreiche Funktionen wahrnahm, unter anderem als Pressereferent, als Vorsitzender des Landesverbandes Niedersachsen und auch als Mitglied im Bundesvorstand.

Sein Wirken in zahlreichen weiteren wichtigen berufspolitischen Gremien auf Länder- und auch Bundesebene war und ist bis heute immer geprägt von den Grundprinzipien des Kampfes für Freiberuflichkeit und deren liberalen Werte, ganz im Sinne einer fairen Kollegialität und auch der Wahrung christlicher Tugenden, was ihm außerordentlich wichtig ist.

Der Dank des FVDZ gilt dem Kollegen und Freund Julius Beischer für sein bisheriges Wirken. Wir wünschen ihm noch viele weitere ereignisreiche und erfüllende Jahre - beruflich und natürlich auch privat.

Dr. Eckhard Jung, FVDZ-Bundesvorstandsmitglied, Praxispartner und langjähriger Weggefährte

\section{Studententag in Frankfurt}

\section{Auslandseinsätze mit Hilfsorganisationen}

Der diesjährige Studententag beim Deutschen Zahnärztetag in Frankfurt am Main spricht auch gestandene Zahnmediziner an. Das gilt ebenso für Zahntechniker und Assistenzpersonal. Denn das Programm unter dem Motto „Famulatur und Auslandseinsatz - Herausforderungen, Möglichkeiten und Realität“ bietet reichhaltige Infos über Auslandseinsätze und zahnmedizinische Hilfsorganisationen.

Studierende erhalten in der Veranstaltung am 12. November, die der Bundesverband der Zahnmedizinstudenten in Deutschland, die Deutsche Gesellschaft für Zahn-, Mund- und Kieferheilkunde und das Gemeinschaftsprojekt mit dem FVDZ, young dentists (yd2), organisieren, wie sie ihre Famulatur mit einem Sinn spendenden Auslandseinsatz verbinden können. Genauso dürfen sich auch junge Zahnärztinnen und Zahnärzte in der Orientierungsphase oder Senioren und Pensionäre angesprochen fühlen.

In Deutschland gibt es verschiedene Organisationen, die im Ausland zahnmedizinisch helfen. Fünf dieser Vereinigungen werden sich in Kurzvorträgen und anschließendem Dialog mit den Teilnehmern vorstellen. Nähere Infos unter www.dtzt.de.

\section{Fachgesellschaft für Parodontologie \\ Vorstand der DG PARO in neuer Zusammensetzung}

Im Vorfeld ihrer Jahrestagung in Würzburg haben die Mitglieder der Deutschen Gesellschaft für Parodontologie (DG PARO) ihren Vorstand neu gewählt. Prof. Dr. Christof Dörfer übernahm turnusgemäß das Amt des Präsidenten. Er wird die nächsten drei Jahre die Geschäfte des Vorstands der Fachgesellschaft führen. Neu nominiert als Präsidentin elect wurde die bisherige Generalsekretärin PD Dr. Bettina Dannewitz. Damit wird zum zweiten Mal in der Geschichte der DG PARO eine geschlechtergemischte Spitze die Interessen der Fachgesellschaft vertreten.

Außerdem im Vorstand: Prof. Dr. med. habil. Dr. h.c. Holger Jentsch als neuer Generalsekretär sowie Prof. Dr. Henrik Dommisch und Dr. Lisa Hierse.

Ausgeschieden aus dem Vorstand sind Dr. Ali Daouk und Prof. Dr. Peter Eickholz, der die DG PARO über mehrere Jahre engagiert und erfolgreich führte.

Weiterhin für die Finanzen zuständig bleibt Schatzmeister Dr. Kai Worch, M.S. (USA). 